\title{
Eco-friendly Flame-Retardant Cellulose Nanofibril Aerogels by Incorporating Sodium Bicarbonate
}

\author{
Muhammad Farooq, $^{\dagger}$ Mika H. Sipponen, ${ }^{\dagger}$ Ari Seppälä, ${ }^{\dagger}$ and Monika Österberg* ${ }^{* \dagger}$ \\ ${ }^{\dagger}$ School of Chemical Engineering, Department of Bioproducts and Biosystems and ${ }^{\ddagger}$ School of Engineering, Department of \\ Mechanical Engineering, Thermodynamics and Combustion Technology, Aalto University, P.O. Box 16300, FI-00076, Aalto, \\ Finland
}

\section{Supporting Information}

ABSTRACT: Cellulose nanofiber (CNF) aerogels offer excellent thermal insulation properties, but high flammability restricts their application. In this study, CNF aerogels were prepared by incorporating sodium bicarbonate (SBC), which effectively improved the fire retardancy without compromising the thermal conductivity of the aerogels, which was only 28 $\mathrm{mW} \mathrm{m} \mathrm{m}^{-1} \mathrm{~K}^{-1}$. The minimum burning velocity of flameretardant aerogels was $0.20 \mathrm{~cm} \mathrm{~s}^{-1}$ at 40 wt \% of SBC, which is significantly lower compared to $5.84 \mathrm{~cm} \mathrm{~s}^{-1}$ of pure CNF aerogels. At the threshold concentration of $20 \mathrm{wt} \%$ SBC, the flame-retardant aerogel demonstrated flameless pyrolysis along with enhanced char formation. SBC additionally provides control over the microporosity and morphology, due to the concentration-dependent formation of lamellar layers during the preparation of aerogels. Overall, this work describes an efficient method for preparing flame-retardant CNF aerogels that could lay the foundation for next-generation bio-based insulation materials.

KEYWORDS: thermal insulation, renewable, polymer, sustainable, freeze-drying

\section{INTRODUCTION}

The development of next-generation bio-based insulation materials is driven by the current environmental strive for sustainability, eco-efficiency, and industrial ecology. ${ }^{1-3}$ Within this realm, efficient utilization of renewable lignocellulose resources holds potential for the development of green products as alternatives to petroleum and mineral-based materials. ${ }^{3,4}$ Cellulose nanofibrils (CNF) have gained renewed interest among the research community in the last two decades. ${ }^{5} \mathrm{CNF}$ aerogels offer impressive properties such as low thermal conductivity, ${ }^{6}$ low thermal expansion, ${ }^{7}$ high strength and elastic modulus, ${ }^{8}$ biocompatibility, and sustainability. ${ }^{9}$ However, flammability ${ }^{10}$ is one of the main drawbacks of aerogels fabricated from CNF, which hinders their use in this application. To meet the strict safety requirements, a considerable improvement in the flame retardancy is mandatory without compromising the insulation properties and thermal and mechanical stabilities. ${ }^{11}$ It is these properties in combination with their microstructure that make $\mathrm{CNF}$ aerogels competitive candidates as effective heat insulators for buildings, domestic appliances, refrigeration equipment, and the automotive sector. ${ }^{12}$

The majority of commercially available flame retardants used in thermal insulation are fossil oil-based, chemically modified organophosphorous, organonitrogen, and halogenated organic compounds. ${ }^{13}$ Especially the latter are highly effective in suppressing flammability, but their harmful impact on the

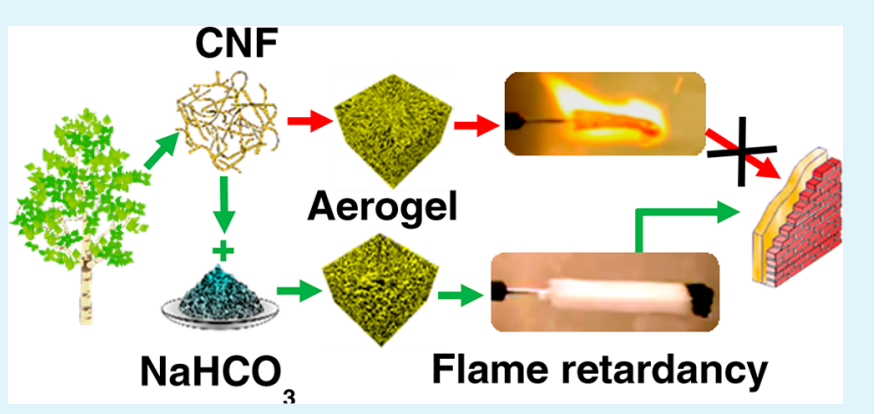

environment and the risk to human health have caused a decline in their acceptance in general and led to legislative restrictions on their use since 2004. ${ }^{14,15}$ Therefore, it is necessary to employ green chemistry principles to develop new environmentally friendly flame-retardant materials.

Han et al. ${ }^{16}$ produced flame-retardant aerogels by in situ synthesis of magnesium hydroxide nanoparticles in cellulose gelled in alkaline urea solution. Resulting aerogels exhibited self-extinguishing behavior within $40 \mathrm{~s}$. However, these composite aerogels exhibited increased thermal conductivity from 56 to $81 \mathrm{~mW} \mathrm{~m}^{-1} \mathrm{~K}^{-1}$ upon higher loading of magnesium hydroxide nanoparticles. A similar approach has recently been adopted by $\mathrm{He}$ et al. ${ }^{17}$ to prepare cellulose-based composite aerogels with improved flame retardancy by utilizing aluminum hydroxide nanoparticles. Wicklein et al. ${ }^{18}$ impregnated a CNF suspension with graphene oxide and clay mineral sepiolite nanorods. Upon freeze-drying, strong anisotropic aerogels were produced, which resisted combustion and exhibited a low thermal conductivity of $15 \mathrm{~mW} \mathrm{~m}^{-1} \mathrm{~K}^{-1}$. Nonetheless, the required use of corrosive acids and oxidants challenges the large-scale production of graphene oxide. ${ }^{19}$

$\mathrm{Li}$ and Wang ${ }^{12}$ coupled polymerization of dopamine and hydrolysis of organosilicon in CNF suspension followed by

Received: March 16, 2018

Accepted: July 23, 2018

Published: July 23, 2018 


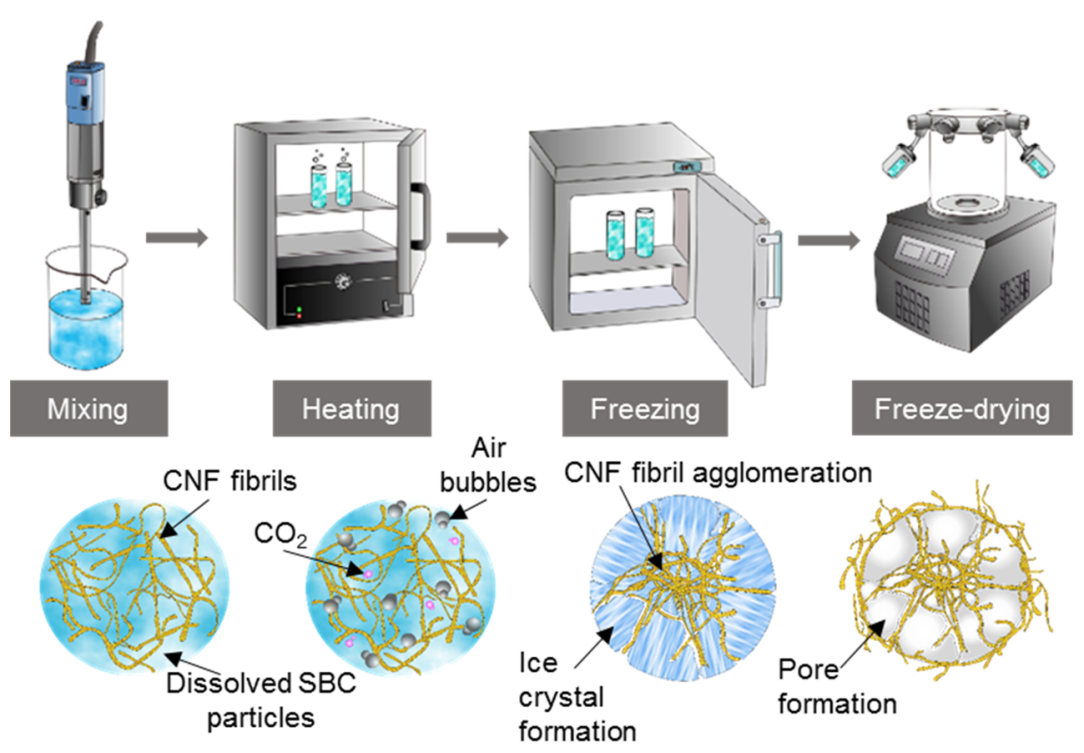

Figure 1. Schematics of the flame-retardant aerogel fabrication procedure and proposed fibrillar structure at each stage.

freeze-drying. The formed aerogels showed self-extinguishing characteristics, but at the trade-off of increased thermal conductivity values. By utilizing the layer-by-layer technique, Köklükaya et al. $^{20}$ deposited cationic chitosan, anionic poly(vinylphosphonic acid), and anionic montmorillonite clay on CNF aerogels. The aerogel coated with five quad layers demonstrated self-extinguishing behavior in a flame test, but the complex fabrication route is the major drawback of this concept. More recently, Yuan and Zhang ${ }^{21}$ prepared composite aerogels by in situ formation of silica nanoparticles in cellulose gel. Supercritical $\mathrm{CO}_{2}$ drying was employed to obtain highly porous flame-retardant aerogels. However, the tetraethoxysilane precursor used in that study has been linked to kidney and nasal mucosa damage. ${ }^{22,23}$

In the present article, we present the preparation of flameretardant aerogels by freeze-drying a colloidal suspension of $\mathrm{CNF}$ in the presence of sodium bicarbonate (SBC) at different concentrations. Our results show that thermal decomposition of sodium bicarbonate to nontoxic gases not only contributes beneficially to the flame-retardant properties, but also offers control over the microporosity of the resulting aerogel.

\section{EXPERIMENTAL SECTION}

2.1. Materials. Sodium bicarbonate (SBC) was obtained from ICN Biomedicals Inc. (Ohio). Poly(vinyl alcohol) (PVA) of MW 22 $\mathrm{kDa}$ was acquired from Fluka Chemie AG. All chemicals were of analytical grade and used without further purification. Nanofibrillated cellulose was produced from hardwood kraft pulp. The pulp was thoroughly washed into sodium form to remove extra electrolytes and control the counterion form. ${ }^{24}$ Fibrillation was performed using the microfluidization process with $\mathrm{M}-110 \mathrm{P}$ processor (Microfluidics, Newton, MA). The pulp was passed one time through chambers of diameters 400 and $200 \mu \mathrm{m}$ and six times through chambers of diameters 400 and $100 \mu \mathrm{m}$ at a pressure of 2000 bar. $^{8}$ The charge density of the pulp used for the preparation of CNF was 0.065 milliequiv $\mathrm{g}^{-1}$, and the $\zeta$-potential value of the corresponding $\mathrm{CNF}$ suspension was $-3 \mathrm{mV}$.

2.2. Preparation of Aerogels. Flame-retardant CNF aerogels were prepared by the freeze-drying procedure. A schematic illustration of the fabrication procedure is presented in Figure 1. Suspensions of CNF $0.5 \%$ by weight were conditioned with sodium bicarbonate at four different weight percentages: $10,20,30$, and $40 \%$ relative to the resulting dry content of the entire mixture. $\mathrm{SBC}$ was dispersed in $\mathrm{CNF}$ suspension using an IKA ULTRA-TURRAX disperser at $10000 \mathrm{rpm}$ for $10 \mathrm{~min}$. The mixture was kept at $100{ }^{\circ} \mathrm{C}$ for $60 \mathrm{~min}$ and freezedried to form aerogels. During exploratory research, it was found that flame-retardant aerogels without heat treatment $\left(100{ }^{\circ} \mathrm{C}\right.$ for $\left.60 \mathrm{~min}\right)$ demonstrate poor mechanical stability. However, the aerogel structure becomes mechanically stable after the heat treatment. Pure CNF aerogel was produced using the abovementioned procedure without the inclusion of sodium bicarbonate. Flame-retardant aerogel samples were coded according to their weight percentage SBC content as CSB10, C-SB20, C-SB30, and C-SB40. Pure CNF was used to prepare the reference aerogel, marked as CNF.

2.3. Morphological Characterization. Structural analysis of CNF aerogels was performed using a JEOL JSM-7500FA analytical field emission scanning electron microscope. Aerogel surfaces and cross sections were sputter-coated with gold/palladium. A secondary electron detector was used for capturing images at $1.5 \mathrm{kV}$. Field emission scanning electron microscopy (FESEM) images were analyzed using the image analysis software FIJI ImageJ (Research Services Branch, NIH, Bethesda, Maryland). ${ }^{25}$

Cylindrical CNF aerogel samples of $10 \mathrm{~mm}$ diameter and $10 \mathrm{~mm}$ length were analyzed by X-ray microtomography (MicroCT). Tomographic radiographs were acquired with Bruker SkyScan 1272 (Kontich, Belgium) at a source voltage of $30 \mathrm{kV}$, a current of $212 \mu \mathrm{A}$, and a pixel size of $14.998 \mu \mathrm{m}$. Exposure time for each projection was set to $212 \mathrm{~ms}$ at a rotation step of $0.4^{\circ}$, along with frame averaging of 5. At least 550 projections, as a stack of $1024 \times 1024$ grayscale bitmap images, containing 16-bit unsigned data were recorded for each sample. A built-in SkyScan program NRecon (Bruker-MicroCT, Kontich, Belgium), which implements the Feldkamp algorithm, was employed for the reconstruction of topography projection images. Three-dimensional (3D) volume rendering and further image processing were performed using the ImageJ software. ${ }^{25}$

2.4. Image Analysis. A total of 300 grayscale MicroCT images for each CNF aerogel were stacked and cropped into $400 \times 400$ pixel square images in ImageJ. The cropped image stacks were subjected to image thresholding by Otsu's method, ${ }^{26}$ which produced a stack of binary images, in which fibril layers and void spaces correspond to white and black areas, respectively.

2.5. Porosity and Pore Size Measurement. The apparent porosity of the pure CNF and flame-retardant aerogels was determined by dividing the total number of void voxels by the sum of black and white voxels present in an image.

$$
\text { Apparent porosity }=\frac{\text { white count }}{\text { white count }+ \text { black count }} \times 100 \%
$$


a

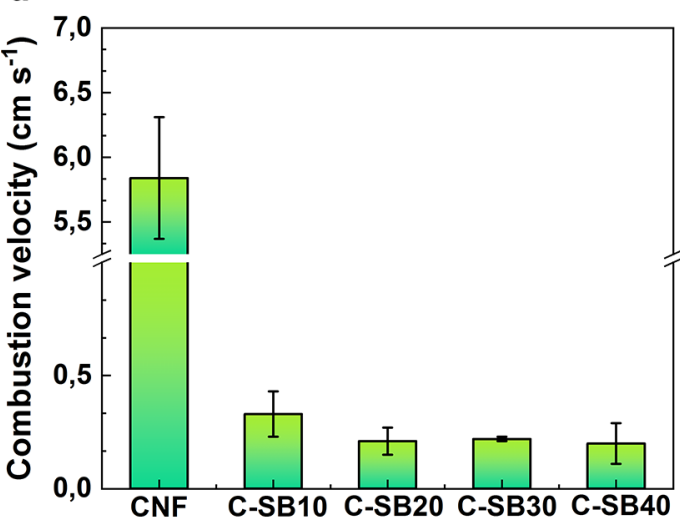

b

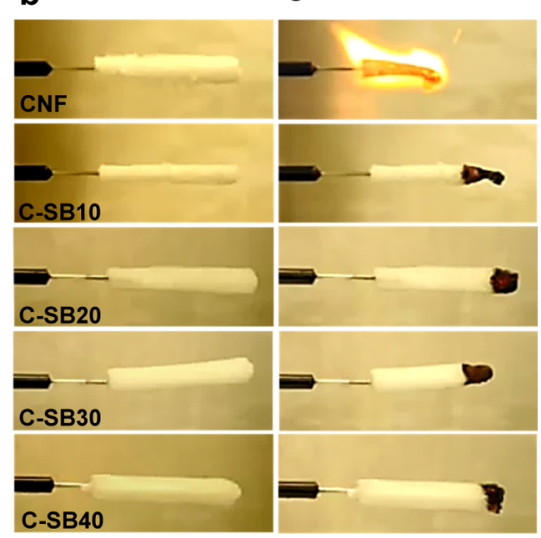

Figure 2. Horizontal flame test of pure CNF aerogel and flame-retardant CNF aerogels containing 10-40 wt \% sodium bicarbonate. (a) Combustion velocities determined from the videos recorded during the flame test. Still images of the aerogels (b) before combustion and (c) after $10 \mathrm{~s}$ combustion, except for pure CNF where the still image is taken after $2 \mathrm{~s}$ of combustion.

The mean apparent porosity of pure and flame-retardant CNF aerogels was calculated from the whole stack of 300-segmented twodimensional (2D) images. Pore size distribution is a critical parameter of porous structures. Five segmented $2 \mathrm{D}$ binary images (slices) from the stack of 300 slices were randomly chosen and subjected to the default particle analyzer algorithm to determine the pore size distribution. To evaluate the length of the fibril layers, ridge detection $^{27}$ was utilized. The slope method was used at default parameters. Five random segmented $2 \mathrm{D}$ binary slices from the stack of 300 were randomly selected for the analysis and mean values were calculated. For comparison, the porosity of the aerogels was also determined by relating the experimental density of the obtained aerogels to the theoretical density of aerogels with $0-40 \mathrm{wt} \%$ of sodium bicarbonate. The dimensions used in the calculation of the experimental densities of cylindrical aerogels were $16 \mathrm{~mm}$ (height) and $12.3 \mathrm{~mm}$ (diameter).

2.6. Chemical Composition. The chemical structure of pure and modified CNF aerogels was analyzed by Fourier transform infrared (FTIR) spectroscopy (Nicolet 380 FTIR Spectrometer by Thermo Fisher Scientific) with $2 \mathrm{~cm}^{-1}$ resolution at room temperature. A total of 30 scans within the range of $400-4000 \mathrm{~cm}^{-1}$ were recorded for each sample. Spectra were adjusted by automatic baseline correction using the built-in OMNIC FTIR software and were normalized relative to the band at $1150 \mathrm{~cm}^{-1}$.

2.7. Thermal Characterization. The thermal properties of pure CNF and modified CNF aerogels were determined using a thermogravimetric analyzer (TA Instruments Q500). Samples were heated from 25 to $600{ }^{\circ} \mathrm{C}$ at a heating rate of $10{ }^{\circ} \mathrm{C} \mathrm{min}-1$ under nitrogen atmosphere at a flow rate of $20 \mathrm{~mL} \min ^{-1}$. Thermal conductivities $(\lambda)$ were measured using a C-Therm thermal conductivity analyzer (C-Therm TCi) by a modified transient source plane technique. Cylindrical samples (diameter $\sim 25 \mathrm{~mm}$, height $\sim 7$ $\mathrm{mm}$ ) of pure CNF and flame-retardant CNF aerogels at four different concentrations of SBC were prepared. Three samples of each aerogel $\left(9 \mathrm{mg}\right.$ ) were measured at $24^{\circ} \mathrm{C}$ from the top and bottom surfaces.

2.8. Flammability Test. The flammability of pure and modified CNF aerogels was tested by igniting the aerogels using a butane burner in a horizontal configuration as described in UL-94 standard. Cylindrical aerogel samples of $1.3 \mathrm{~cm} \times 6 \mathrm{~cm}$ were held in the horizontal position using metal clamps and ignited endwise. This horizontal flame test was repeated three times. The whole combustion process was recorded by a digital camera, and the combustion velocity was measured at $10 \mathrm{~s}$.

2.9. Polymer and Moisture Absorption. Aerogel sections (1.5 $\mathrm{cm}$ ) were soaked in $15 \mathrm{wt} \%$ aqueous poly(vinyl alcohol) (PVA) solution until saturation absorption. Absorption capacity was calculated as the ratio of weight increase to the initial weight. Moisture absorption was evaluated by exposing aerogel sections (1.5 $\mathrm{cm})$ to $80 \%$ relative humidity for $48 \mathrm{~h}$. Moisture uptake was determined from the weight gain relative to the weight of aerogels that were preconditioned at $50 \%$ relative humidity.

\section{RESULTS AND DISCUSSION}

3.1. Flame-Retarding Properties of SBC-CNF Aerogels. CNF aerogels were prepared using sodium bicarbonate as a green flame-retarding agent. The flame-retarding performance of pure and SBC-containing CNF aerogels was determined by a horizontal combustion test (Figure 2b). Pure CNF aerogels ignited extremely quickly and engulfed in flames in $<1 \mathrm{~s}$. The average combustion velocity of pure $\mathrm{CNF}$ aerogel was $5.84 \mathrm{~cm} \mathrm{~s}^{-1}$. In contrast, SBC-CNF aerogels exhibited a distinctly lower burning rate. Already $10 \mathrm{wt} \%$ of SBC (C-SB10) had a considerable flame-retarding effect compared to the pure CNF aerogel. The burning velocity of C-SB10 was $0.33 \mathrm{~cm} \mathrm{~s}^{-1}$ along with a self-extinguishing behavior after $5 \mathrm{~s}$ of initial burning. The slow, flameless combustion partially consumed the sample, leaving behind a partly preserved aerogel and a charred residue. At higher concentrations of SBC, the flame-retardant aerogels (C-SB20, C-SB30, and C-SB40) self-extinguished as the flame source was removed. A further decrease in the combustion velocity was also observed; however, after $10 \mathrm{~s}$ of burning, the combustion velocity did not change notably among C-SB20, C-SB30, and C-SB40 samples. Combustion velocity data and the burning behavior revealed that the aerogel at $20 \mathrm{wt} \%$ SBC shows optimum flame-retarding properties in terms of selfextinguishing behavior and pyrolysis (Figure 2c). Overall, the incorporation of SBC efficiently circumvents the flammability of CNF aerogels, which was probably due to the release of carbon dioxide and water upon heat-induced decomposition. To further confirm this hypothesis, the physical, chemical, and thermal properties of the aerogels were determined more in detail.

3.2. Morphology and Chemical Structure of the Aerogels. Porosity is an important feature of an aerogel structure since it determines the effective volume of air in the matrix. X-ray MicroCT and image processing were used to evaluate the apparent porosity values. X-ray MicroCT has a limited spatial resolution, which hinders its ability to capture the whole range of pore sizes in a sample volume. However, we found that the porosity values obtained from MicroCT imaging are useful for explaining observations on morpho- 

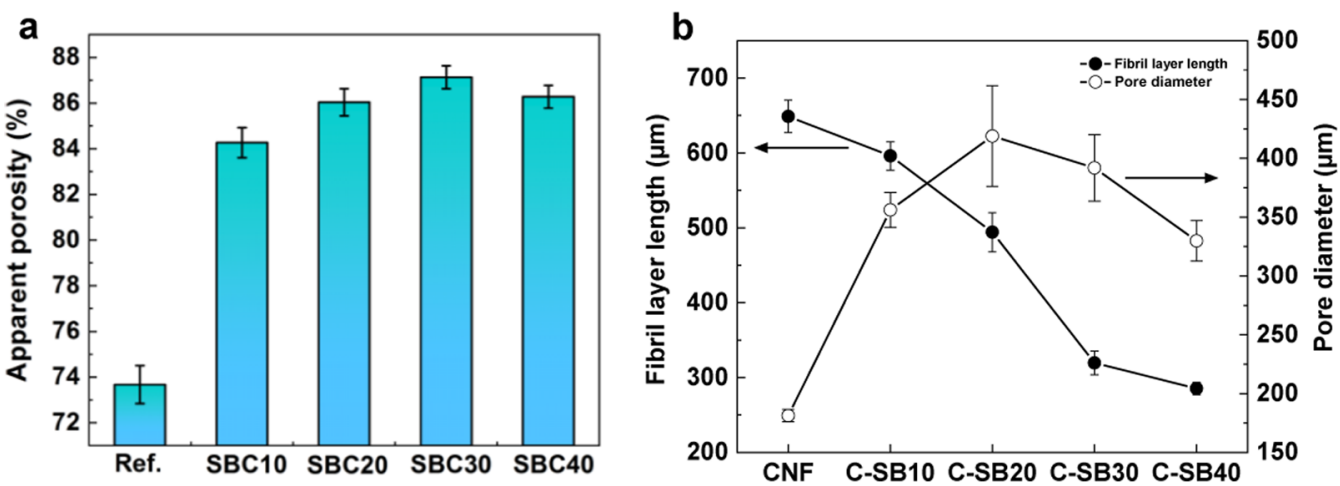

Figure 3. Effect of sodium bicarbonate on the morphology of CNF aerogels. (a) Apparent porosity and (b) mean pore diameter and mean fibril layer length. Values of apparent porosity, pore diameter, and mean fibril length were obtained from image analysis of MicroCT data.
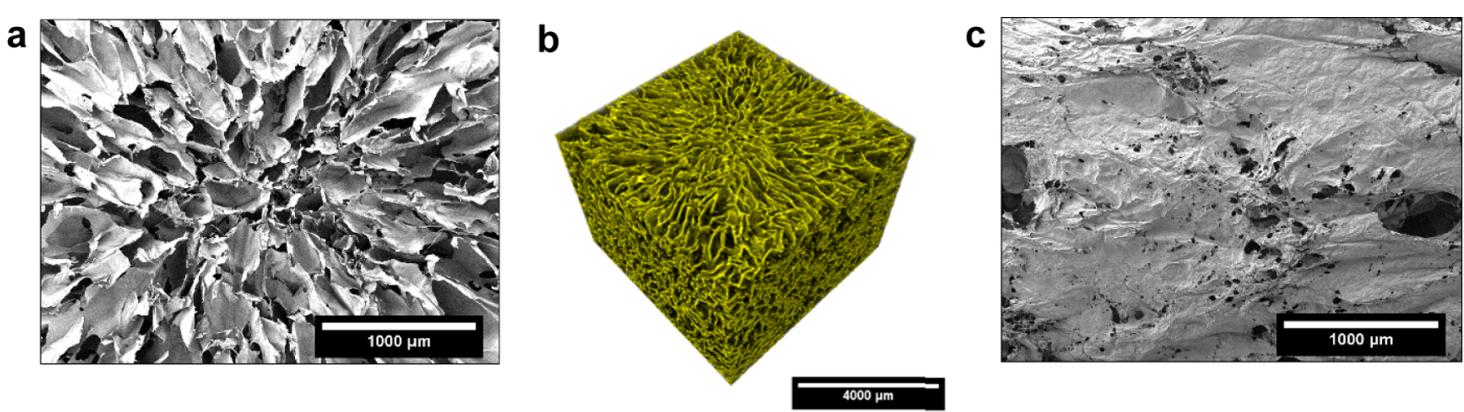

d
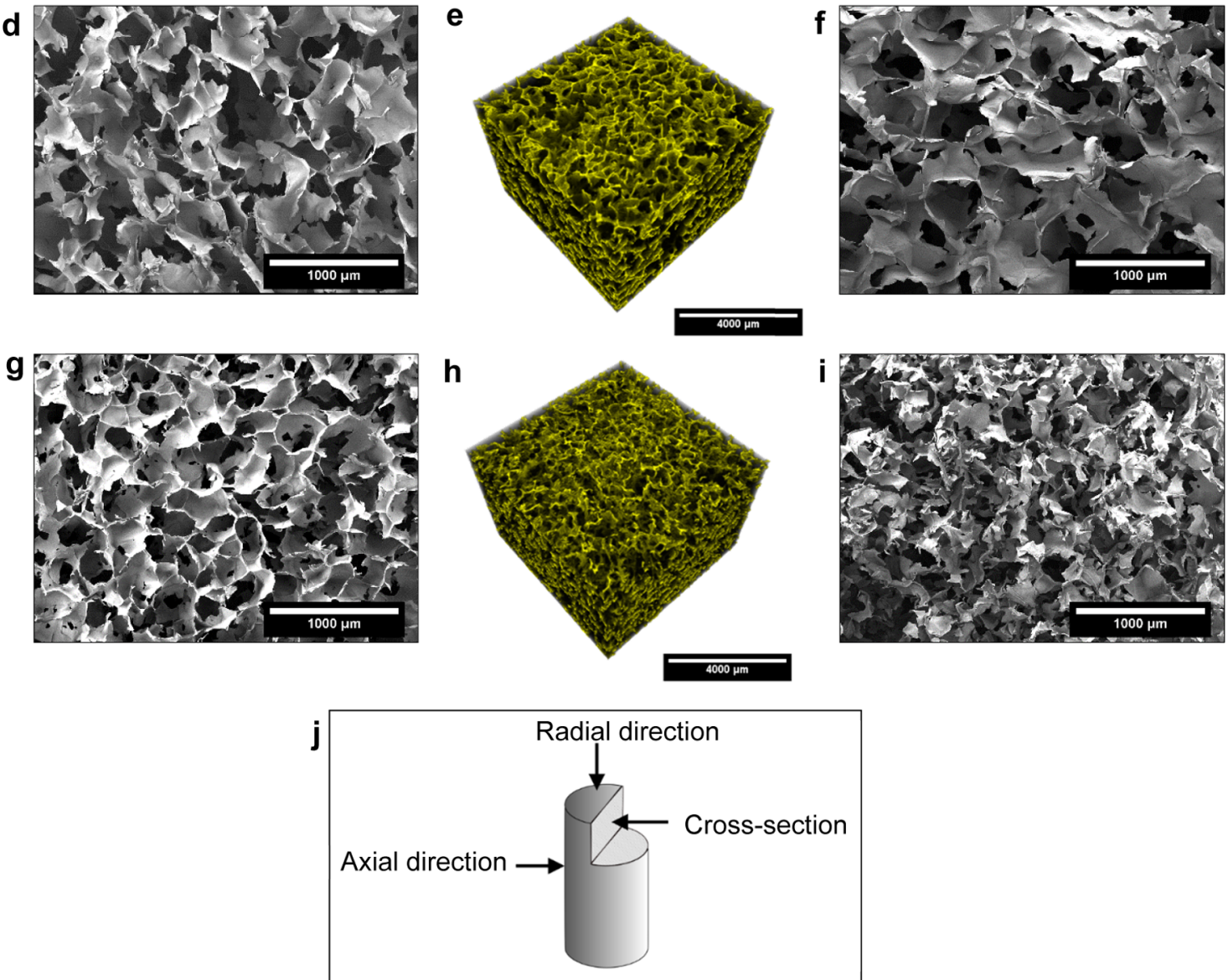

Figure 4. Morphological appearance of pure CNF aerogel and SBC-containing aerogels. $(a-c)$ Pure CNF aerogel, $(d-f) C-S B 10$, and $(g-i) C$ SB30. (b, e, h) 3D volumetric rendering from MicroCT data. (a, d, g) and (c, f, i) FESEM images in radial and axial directions, respectively. (j) Aerogel sample sketch detailing cross-section and radial and axial directions. 
logical differences between the flame-retardant aerogel samples.

The effect of SBC addition on the apparent porosity is illustrated in Figure 3a. Apparent porosity increased from 74\% of pure CNF aerogel to $84 \%$ when the SBC concentration was 10 wt \%. Further increase in the SBC concentration showed only a minor influence on the apparent porosity, and the maximum value of $87 \%$ was obtained at $30 \mathrm{wt} \% \mathrm{SBC}$. The apparent porosity of pure CNF aerogel is lower than the reported density-based porosities ${ }^{28,18}$ of CNF aerogels prepared using the freeze-drying procedure. However, density-based porosities for all of our aerogels shown in the Supporting Information Table S2 were $>99.5 \%$, in agreement with published data. ${ }^{18}$ The deviation between apparent and density-based porosities is therefore anticipated to result from macroscale changes due to the heating step during the aerogel preparation, which would promote the aggregation of nanofibrils, thus causing layer formation and reduction in the apparent porosity.

The pure CNF aerogel had the lowest average pore diameter of $181 \mu \mathrm{m}$, whereas among the flame-retardant CNF aerogels, C-SB20 exhibited the highest average pore diameter of $419 \mu \mathrm{m}$ (Figure 3b.) Furthermore, SBC had a disruptive effect on the fibril layers, decreasing the fibril layer length from $615 \mu \mathrm{m}$ in the pure CNF to $494 \mu \mathrm{m}$ in C-SB20 and further to $319 \mu \mathrm{m}$ in C-SB30 (Figure 3b). These observations were supported by the FESEM image analysis and volumetric rendering of MicroCT data (Figure 4).

Pure CNF aerogel contained an organized fibril layer structure, which converges toward the center of the aerogel (Figure 4a). Fibrils are highly interconnected, hence causing a dense aerogel structure. The outer surface of pure CNF aerogel exhibited a smooth $2 \mathrm{D}$ sheetlike structure (Figure 4c) with a few pores present on the surface. Important differences compared to the pure CNF aerogel were observed already at the lowest sodium bicarbonate dosing. The smooth outer surface of the pure CNF aerogel became disrupted in C-SB10 aerogel due to the emergence of large pores of diameter 356 $\mu \mathrm{m}$ (Figure 4f). The radial micrograph (Figure $4 \mathrm{~d}$ ) also depicted dissimilarities in terms of surface and pore morphology with respect to pure CNF aerogel (Figure 4a). C-SB30 exhibited a honeycomb-like structure in the radial direction (Figure $4 \mathrm{~g}$ ), forming a cellular structure with a wall thickness of approximately 3-5 $\mu \mathrm{m}$. The outer surface of CSB30 (Figure 4i) displayed a disrupted fibril layer structure with a smaller pore diameter compared to C-SB10. At the highest concentration of SBC (C-SB40), the aerogel structure collapsed, forming a microstructure composed of smaller pores with a pore diameter of $330 \mu \mathrm{m}$ (Supporting Information Figure S1). Importantly, regardless of such high loading of SBC, the connectivity of the fibril layer structure was still preserved, maintaining physical integrity of the aerogel.

During the freeze-drying process, cellulose nanofibrils align laterally and form layer structures of varying lengths due to the diffusion forces or interfibrillar hydrogen bonding. ${ }^{29}$ The SBC content played a decisive role in the resulting morphology of aerogels. Partial decomposition of sodium bicarbonate resulting in the formation of carbon dioxide and the presence of sodium carbonate or bicarbonate crystals near cellulose nanofibrils may have hindered the interfibrillar attraction, hence causing disruption of the fibril layer structure. This likely explains the aforementioned differences observed in the apparent porosity and surface morphology of the CNF aerogels (Figure 3).

FTIR spectroscopy was used to determine the presence of sodium bicarbonate and sodium carbonate residues and their possible interaction with cellulose within the aerogels. Typical absorptions of the cellulose backbone ${ }^{30}\left(v_{\mathrm{O}-\mathrm{H}} 3340 \mathrm{~cm}^{-1}\right.$, $v_{\mathrm{C}-\mathrm{H}} 2904 \mathrm{~cm}^{-1}, 1429 \mathrm{~cm}^{-1}, v_{\mathrm{H}-\mathrm{O}-\mathrm{H}} 1641 \mathrm{~cm}^{-1}, v_{\mathrm{C}-\mathrm{C}} 1160$ $\mathrm{cm}^{-1}, v_{\mathrm{C}-\mathrm{O}-\mathrm{C}} 1054 \mathrm{~cm}^{-1}, v_{\beta \text {-linkage }} 896 \mathrm{~cm}^{-1}$ ) were observed (Figure 5).

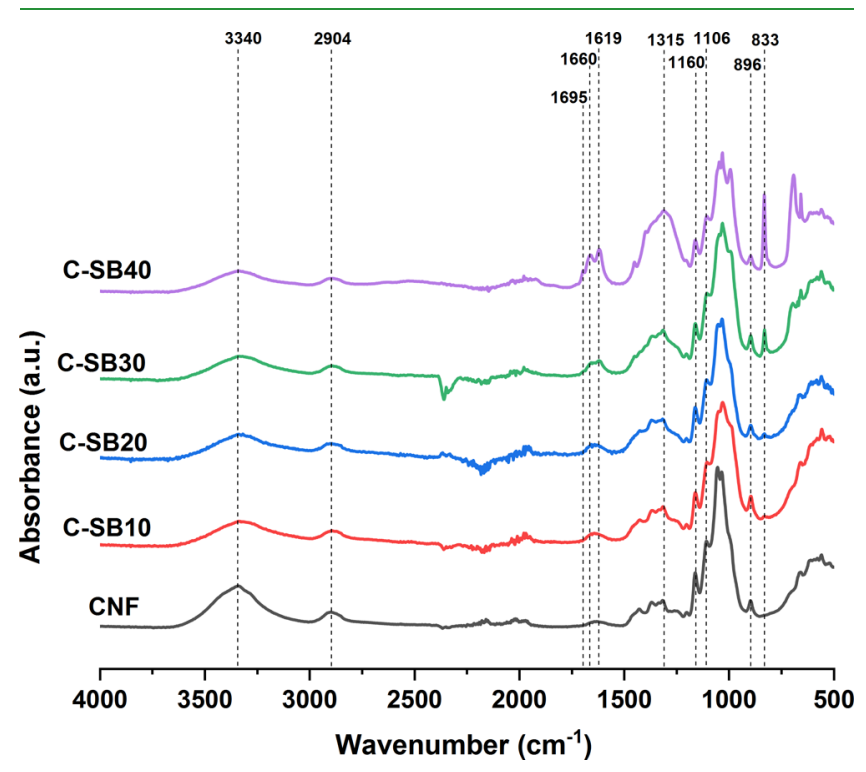

Figure 5. FTIR spectra of pure and flame-retardant CNF aerogels.

In the wavelength region of $2000-4000 \mathrm{~cm}^{-1}$, the normalized spectra of pure $\mathrm{CNF}$ and modified aerogels exhibited similar characteristic bands; however, at lower wavenumbers, a new absorption band at $833 \mathrm{~cm}^{-1}$ emerged in the spectra of the SBC-containing aerogels. This band is associated with the $\mathrm{CO}_{2}$ bending vibrations present in $\mathrm{NaHCO}_{3} .{ }^{31}$ The height of this band increased with increasing concentration of SBC (Supporting Information Figure S2). Furthermore, three consecutive bands at 1619, 1660, and 1695 $\mathrm{cm}^{-1}$ in the IR spectra of C-SB40 were assigned to the $\mathrm{CO}_{2}$ out-of-phase stretching in sodium bicarbonate. ${ }^{31}$ The emergence of new absorption bands confirmed the expected presence of SBC in the flame-retardant aerogels. The products of thermal decomposition of SBC are sodium carbonate, water, and carbon dioxide; however, no characteristic signal was observed for sodium carbonate. Furthermore, the comparison of theoretical and calculated SBC content in the flame retardant aerogels indicated only limited decomposition in the heating stage prior to aerogel formation (Supporting Information Figure S5).

3.3. Thermal Properties of Flame-Retardant CNF Aerogels. Considering the heat insulation application, thermal stability of flame-retardant aerogels is of great importance. ${ }^{26}$ Thermal analysis can also assist in understanding the mechanisms of fire resistance. Thermal analyses were performed in nitrogen atmosphere over the temperature range of $25-600{ }^{\circ} \mathrm{C}$. Addition of SBC in flame-retardant aerogels caused multiple changes in their thermal characteristics compared to those of pure CNF aerogel (Figure 6). The major decomposition peak of flame-retardant aerogels shifted toward lower temperatures and the decomposition peak 

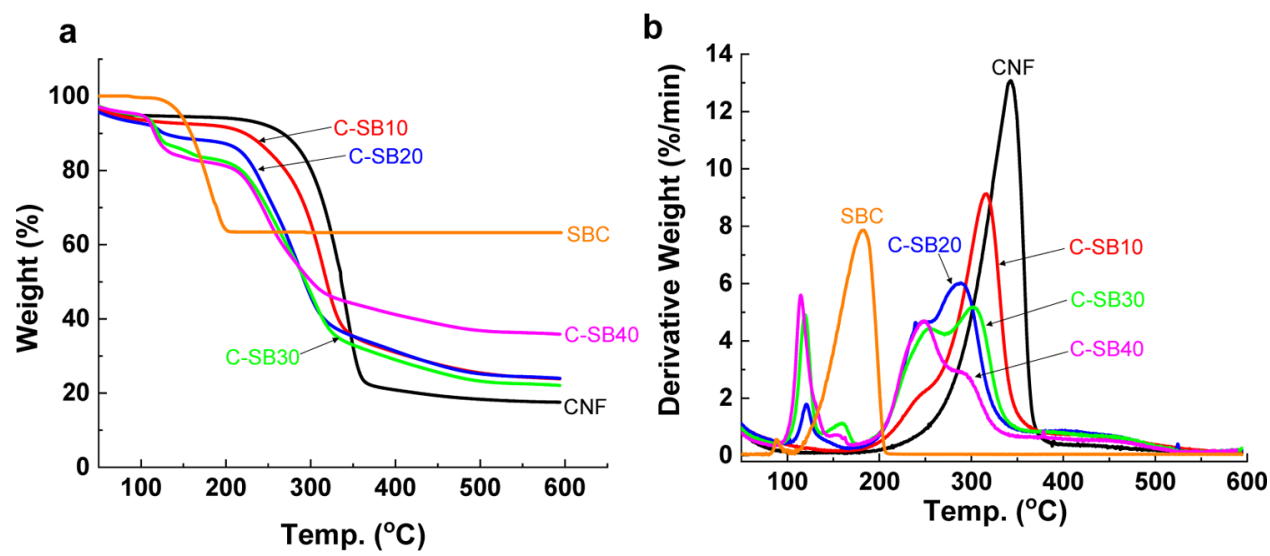

Figure 6. Thermogravimetric analysis (TGA) (a) and derivative thermogravimetry (DTG) (b) curves of sodium bicarbonate, pure CNF aerogel, and flame-retardant CNF aerogels.
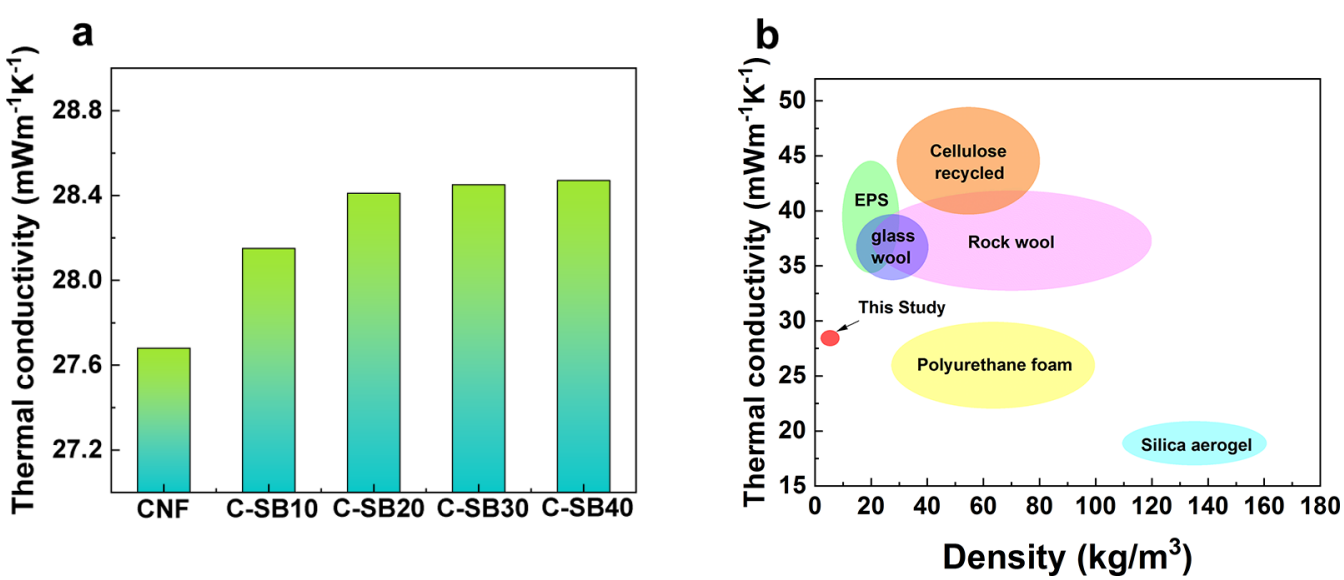

Figure 7. Thermal conductivities of aerogels. (a) Thermal conductivity $(\lambda)$ values of pure and flame-retardant CNF aerogels. (b) Ashby plot of thermal conductivity versus density for commonly used insulation materials including this study and values obtained from ref 36 .

became less pronounced, which support the argument that sodium bicarbonate is an effective flame retardant.

The TGA image of pure CNF aerogel consisted of a single cellulose decomposition ${ }^{32}$ phase in the temperature range of 306-359 ${ }^{\circ} \mathrm{C}$, whereas the major decomposition peak for CSB10 shifted toward lower temperatures with an onset temperature of $277{ }^{\circ} \mathrm{C}$. At the highest concentration of SBC, the C-SB40 aerogel exhibited an onset temperature of $223{ }^{\circ} \mathrm{C}$ and maximum decomposition at $262{ }^{\circ} \mathrm{C}$. These changes likely result from the decomposition of sodium bicarbonate, as also indicated by the emergence of a new peak at $115-121{ }^{\circ} \mathrm{C}$ in the DTG images of C-SB20, C-SB30, and C-SB40 (Figure 6b). The amount of residue generated by C-SB40 is $36 \%$, which is $19 \%$ more than that of the pure CNF aerogel. Sodium bicarbonate exhibited a single decomposition peak with an onset temperature of $144{ }^{\circ} \mathrm{C}$. To assess possible decomposition of sodium bicarbonate during the heating stage of aerogel preparation, the remaining SBC content was determined from the TGA data at $200{ }^{\circ} \mathrm{C}$ and compared to the theoretical input values (Supporting Information Table S4 and Figure S5). The data showed that the theoretical and quantified SBC contents were essentially similar, indicating insignificant losses of SBC. The amount of residue generated during pure sodium bicarbonate decomposition is $63 \%$, which explains the higher residue in flame-retardant aerogels. Flameretardant aerogels displayed multiple peaks in DTG curves. It is considered that the multiple peaks displayed by the flame- retardant aerogels represent the exothermic changes during the decomposition and gas liberation process. ${ }^{33}$ Values of the onset decomposition temperature $\left(T_{\text {on }}\right)$, the temperature at the maximum degradation rate $\left(T_{\mathrm{d}}\right)$, weight loss at each stage, and residue for pure and flame-retardant aerogel samples are tabulated in Supporting Information Table S1. The presence of sodium bicarbonate works as a green flame retardant in two separate ways. First, it absorbs heat to decompose, and once decomposed, it liberates carbon dioxide and water. The $\mathrm{CO}_{2}$ deprives the supply of oxygen required during the burning process, while water requires energy to vaporize, hence consuming more energy. ${ }^{33}$

The insulation performance of pure CNF and flameretardant aerogels is shown in Figure 7a. Importantly, the thermal conductivity remained practically unchanged upon addition of sodium bicarbonate, with the maximum difference being only $0.8 \mathrm{~mW} \mathrm{~m}^{-1} \mathrm{~K}^{-1}$ between the pure CNF aerogel $\left(27.7 \mathrm{~mW} \mathrm{~m}^{-1} \mathrm{~K}^{-1}\right)$ and C-SB40 $\left(28.5 \mathrm{~mW} \mathrm{~m}^{-1} \mathrm{~K}^{-1}\right)$. This shows that despite the extensive improvement in the flameretardant properties upon addition of SBC, the thermal insulating properties were not hampered, which is rather unique for modified CNF aerogels. In fact, the thermal conductivity values presented in this study are superior to those presented in the literature. For CNF-based flameretardant aerogels, typical previously reported values of thermal conductivity vary between 30 and $80 \mathrm{~mW} \mathrm{~m}^{-1}$ $\mathrm{K}^{-1} \cdot{ }^{16,12,34,3516,12,34,35}$ The lowest reported ${ }^{18}$ thermal con- 
ductivity value for CNF-based flame-retardant nanocomposites is $15 \mathrm{~mW} \mathrm{~m}^{-1} \mathrm{~K}^{-1}$. This was measured in the radial direction, while the thermal conductivity for these composites in the axial direction was $170 \pm 3 \mathrm{~mW} \mathrm{~m}^{-1} \mathrm{~K}^{-1}$. Here, it is important to mention that the thermal conductivity values in the present study were evaluated in the axial direction, which are significantly lower compared to the reported value of CNFbased flame-retardant nanocomposites. ${ }^{18}$

The Ashby plot presented in Figure $7 \mathrm{~b}$ displays thermal conductivity of various thermal insulation materials as a function of density. It is evident that the heat insulating properties of the flame-retardant aerogels reported in the present work are significantly better than those of conventional thermal insulating materials, such as glass wool, expanded polystyrene (EPS), polyurethane foams, and rock wool. Among these thermal insulating materials, EPS is largely employed in construction, but due to its high flammability, organohalogen flame retardants, such as hexabromocyclododecane, bisallylether of tetrabromobisphenol A, and tribromophenyl allyl ester, are used. However, these halogenated flame-retarding agents produce harmful substances during combustion, causing concerns to human health and environment. ${ }^{37}$

Silica aerogels have the lowest thermal conductivity values ranging between 17 and $21 \mathrm{~mW} \mathrm{~m}^{-1} \mathrm{~K}^{-1}$; however, the manufacturing process of silica aerogels involves the use of hazardous solvents, a supercritical drying process, and high pressure and temperature. ${ }^{38}$ Moreover, silica aerogels also suffer from fragility and brittleness, which limit their applications in many fields. ${ }^{39,40}$ In contrast, the thermal conductivities of CNF-based flame-retardant aerogels prepared in this work are superior to the expanded polystyrene (EPS) and comparable to polyurethane insulation foam, both of which occupy considerable market shares today.

3.4. Polymer and Moisture Absorption. To assess the feasibility of flame-retarding aerogels for polymer-based nanocomposites manufacturing, the polymer (PVA) absorption capacity of pure $\mathrm{CNF}$ and flame-retardant aerogels was calculated by soaking aerogels in aqueous $15 \mathrm{wt} \%$ PVA solution. The polymer absorption capacity was directly related to the CNF content (Supporting Information Figure S3). The pure CNF aerogel showed the highest polymer absorption capacity, while the lower mass fraction of nanocellulose in the flame-retardant aerogels reduced the polymer absorption. This can be a result of lower number of exposed hydroxyl groups on the surface of aerogels. Hydroxyl groups could form hydrogen bonds with PVA molecules; hence, the availability of hydroxyl groups would influence PVA absorption.

From the moisture absorption data, it was found that increased concentration of sodium bicarbonate led to an increment in the moisture uptake (\%) of the flame-retardant aerogels. Pure CNF aerogel exhibits a moisture uptake of $6.2 \%$, whereas C-SB40 aerogel demonstrates a moisture uptake value of $16.7 \%$ (Supporting Information Figure S4).

\section{CONCLUSIONS}

Flame-retardant and heat insulating CNF aerogels were successfully fabricated by incorporating sodium bicarbonate as a flame-retarding agent. This facile and environmentally friendly approach produced physically stable aerogels with high porosity, low thermal conductivity, and considerable flame retardancy. Different concentrations of sodium bicarbonate altered the microstructure of flame-retardant aerogels, providing control over the aerogel morphology. This low- cost method reinforces the use of readily available renewable materials without the need for harmful additives. Furthermore, the flame-retardant properties of the CNF aerogels in this study make them well-suited candidates for thermal insulation, not only due to their bio-based origin and competitive thermal conductivity values, but also due to the harmless gases produced during the flameless pyrolysis. The developed method can pave the way for the production of green insulating aerogels for replacing oil-based insulation materials in various sectors.

\section{ASSOCIATED CONTENT}

\section{Supporting Information}

The Supporting Information is available free of charge on the ACS Publications website at DOI: 10.1021/acsami.8b04376.

Additional FESEM images of pure CNF and flameretardant aerogels (Figure S1); FTIR peak ratio $(833: 896)$ as a function of SBC concentration (Figure S2); polymer absorption capacity of pure CNF and flame-retardant CNF aerogels submerged in 15 wt \% PVA solution during $2 \mathrm{~min}$ (Figure S3); moisture absorption of pure $\mathrm{CNF}$ and flame-retardant aerogels at $80 \%$ RH (Figure S4); comparison of theoretical and calculated SBC content of flame-retardant CNF aerogels (Figure S5); comparison of theoretical and experimental densities of pure and flame-retardant CNF aerogels (Figure S6); values of the onset decomposition temperature $\left(T_{\text {on }}\right)$, temperature at the maximum degradation rate $\left(T_{\mathrm{d}}\right)$, and weight loss at each stage and residue for sodium bicarbonate, pure $\mathrm{CNF}$, and flame-retardant aerogel samples (Table S1); porosity values of pure and flame-retardant CNF aerogels (Table S2); TGA mass balance comparison of theoretical and calculated SBC content at $200{ }^{\circ} \mathrm{C}$ of flame-retardant CNF aerogels (Table S4); experimental and theoretical densities of pure and flame-retardant CNF aerogels (Table S5) (PDF)

Videos of combustion of pure CNF and flame-retardant aerogels (Video S1); combustion of pure CNF aerogel (AVI)

Combustion of C-SB10 aerogel (Video S2) (AVI)

Combustion of C-SB30 aerogel (Video S3) (AVI)

Videos of the $3 \mathrm{D}$ porous structure of pure CNF and flame-retardant aerogels (Video S4); visualization of 3D porous structure of pure CNF aerogel (AVI)

Visualization of 3D porous structure of C-SB10 aerogel (Video S5) (AVI)

Visualization of 3D porous structure of C-SB30 aerogel (Video S6) (AVI)

\section{- AUTHOR INFORMATION}

\section{Corresponding Author}

*E-mail: monika.osterberg@aalto.fi. Tel: +358505497218. ORCID

Mika H. Sipponen: 0000-0001-7747-9310

Monika Österberg: 0000-0002-3558-9172

\section{Author Contributions}

M.F. conducted the experiments and analyzed the data in collaboration with M.H.S. and M.Ö. A.S. helped with performing thermal conductivity measurements. M.F. wrote the manuscript with inputs from all of the authors. All of the 
authors discussed the results and read and approved the manuscript.

\section{Notes}

The authors declare no competing financial interest.

\section{ACKNOWLEDGMENTS}

The authors acknowledge the provision of facilities and technical support by Aalto University at OtaNano-Nanomicroscopy Center (Aalto-NMC) and Bioeconomy facilities and University of Helsinki for X-ray microtomography (MicroCT) imaging system. M.H.S. and M.Ö. acknowledge financial support from the Academy of Finland (postdoctoral and project grants 296547 and 278279, respectively). M.F. acknowledges Aalto CHEM for the financial support.

\section{REFERENCES}

(1) Zhu, H.; Luo, W.; Ciesielski, P. N.; Fang, Z.; Zhu, J. Y.; Henriksson, G.; Himmel, M. E.; Hu, L. Wood-Derived Materials for Green Electronics, Biological Devices, and Energy Applications. Chem. Rev. 2016, 116, 9305-9374.

(2) Faruk, O.; Bledzki, A. K.; Fink, H.-P.; Sain, M. Progress Report on Natural Fiber Reinforced Composites. Macromol. Mater. Eng. 2014, 299, 9-26.

(3) Dale, B. E. 'Greening' the Chemical Industry: Research and Development Priorities for Biobased Industrial Products. J. Chem. Technol. Biotechnol. 2003, 78, 1093-1103.

(4) Carole, T. M.; Pellegrino, J.; Paster, M. D. Opportunities in the Industrial Biobased Products Industry. Appl. Biochem. Biotechnol. 2004, 115, 871-885.

(5) Lavoine, N.; Bergstrom, L. Nanocellulose-Based Foams and Aerogels: Processing, Properties, and Applications. J. Mater. Chem. A 2017, 5, 16105-16117.

(6) Uetani, K.; Hatori, K. Thermal Conductivity Analysis and Applications of Nanocellulose Materials. Sci. Technol. Adv. Mater. 2017, 18, 877-892.

(7) Carosio, F.; Kochumalayil, J.; Cuttica, F.; Camino, G.; Berglund, L. Oriented Clay Nanopaper from Biobased ComponentsMechanisms for Superior Fire Protection Properties. ACS Appl. Mater. Interfaces 2015, 7, 5847-5856.

(8) Österberg, M.; Vartiainen, J.; Lucenius, J.; Hippi, U.; Seppälä, J.; Serimaa, R.; Laine, J. A Fast Method to Produce Strong NFC Films as a Platform for Barrier and Functional Materials. ACS Appl. Mater. Interfaces 2013, 5, 4640-4647.

(9) Klemm, D.; Heublein, B.; Fink, H.-P.; Bohn, A. Cellulose: Fascinating Biopolymer and Sustainable Raw Material. Angew. Chem., Int. Ed. 2005, 44, 3358-3393.

(10) Dorez, G. Thermal and Fire Behavior of Natural Fibers/PBS Biocomposites. Polym. Degrad. Stab. 2013, 98, 87-95.

(11) Wang, D. Y. Novel Fire Retardant Polymers and Composite Materials; Elsevier: Amsterdam, 2016.

(12) Li, Y.; Wang, B.; Sui, X.; Xu, H.; Zhang, L.; Zhong, Y.; Mao, Z. Facile Synthesis of Microfibrillated Cellulose/Organosilicon/Polydopamine Composite Sponges with Flame Retardant Properties. Cellulose 2017, 24, 3815-3823.

(13) Costes, L.; Laoutid, F.; Brohez, S.; Dubois, P. Bio-Based Flame Retardants: When Nature Meets Fire Protection. Mater. Sci. Eng., $R$ 2017, 117, 1-25.

(14) Anon. Flame retardants: European Union Risk Assessments Update. Plast. Addit. Compd. 2004, 6, 26-29.

(15) Hale, R. C.; La Guardia, M. J.; Harvey, E.; Gaylor, M. O.; Mainor, T. M. Brominated Flame Retardant Concentrations and Trends in Abiotic Media. Chemosphere 2006, 64, 181-186.

(16) Han, Y.; Zhang, X.; Wu, X.; Lu, C. Flame Retardant, Heat Insulating Cellulose Aerogels from Waste Cotton Fabrics by in Situ Formation of Magnesium Hydroxide Nanoparticles in Cellulose Gel Nanostructures. ACS Sustainable Chem. Eng. 2015, 3, 1853-1859.
(17) He, C.; Huang, J.; Li, S.; Meng, K.; Zhang, L.; Chen, Z.; Lai, Y. Mechanically Resistant and Sustainable Cellulose-Based Composite Aerogels with Excellent Flame Retardant, Sound-Absorption, and Superantiwetting Ability for Advanced Engineering Materials. ACS Sustainable Chem. Eng. 2018, 6, 927-936.

(18) Wicklein, B.; Kocjan, A.; Salazar-Alvarez, G.; Carosio, F.; Camino, G.; Antonietti, M.; Bergström, L. Thermally Insulating and Fire-Retardant Lightweight Anisotropic Foams Based on Nanocellulose and Graphene Oxide. Nat. Nanotechnol. 2015, 10, 277.

(19) Lowe, S. E.; Zhong, Y. L. Challenges of Industrial-Scale Graphene Oxide Production. In Graphene Oxide: Fundamentals and Applications; Dimiev, A. M., Eigler, S., Eds.; John Wiley \& Sons: West Sussex, United Kingdom, 2016; pp 410-431.

(20) Köklükaya, O.; Carosio, F.; Wågberg, L. Superior FlameResistant Cellulose Nanofibril Aerogels Modified with Hybrid Layerby-Layer Coatings. ACS Appl. Mater. Interfaces 2017, 9, 2908229092.

(21) Yuan, B.; Zhang, J.; Mi, Q.; Yu, J.; Song, R.; Zhang, J. Transparent Cellulose-Silica Composite Aerogels with Excellent Flame Retardancy via an in Situ Sol-Gel Process. ACS Sustainable Chem. Eng. 2017, 5, 11117-11123.

(22) Nakashima, H.; Omae, K.; Sakai, T.; Yamazaki, K.; Sakurai, H. Acute and Subchronic Inhalation Toxicity of Tetraethoxysilane (TEOS) in Mice. Arch. Toxicol. 1994, 68, 277-283.

(23) Nakashima, H. Time Course of Effects of Tetraethoxysilane (TEOS) on the Kidney and Blood Silicon Concentration in Mice. Arch. Toxicol. 1994, 69, 59-64.

(24) Swerin. Deswelling of Hardwood Kraft Pulp Fibers by Cationic Polymers the Effect on Wet Pressing and Sheet Properties. Nord. Pulp Pap. Res. J. 1990, 05, 188-196.

(25) Schindelin, J.; Arganda-Carreras, I.; Frise, E.; Kaynig, V.; Longair, M.; Pietzsch, T.; Preibisch, S.; Rueden, C.; Saalfeld, S.; Schmid, B.; Tinevez, J.-Y.; White, D. J.; Hartenstein, V.; Eliceiri, K.; Tomancak, P.; Cardona, A. Fiji: An Open-Source Platform for Biological-Image Analysis. Nat. Methods 2012, 9, 676.

(26) Otsu, N. A Threshold Selection Method from Gray-Level Histograms. IEEE Trans. Syst. Man Cybern. 1979, 9, 62-66.

(27) Steger, C. An Unbiased Detector of Curvilinear Structures. IEEE Trans. Pattern Anal. Mach. Intell. 1998, 20, 113-125.

(28) Pääkkö, M.; Vapaavuori, J.; Silvennoinen, R.; Kosonen, H.; Ankerfors, M.; Lindstrom, T.; Berglund, L. A.; Ikkala, O. Long And Entangled Native Cellulose I Nanofibers Allow Flexible Aerogels and Hierarchically Porous Templates for Functionalities. Soft Matter 2008, 4, 2492-2499.

(29) Peng, Y.; Gardner, D. J.; Han, Y. Drying Cellulose Nanofibrils: In Search of a Suitable Method. Cellulose 2012, 19, 91-102.

(30) Chen, W.; Li, Q.; Wang, Y.; Yi, X.; Zeng, J.; Yu, H.; Liu, Y.; Li, J. Comparative Study of Aerogels Obtained from Differently Prepared Nanocellulose Fibers. ChemSusChem 2014, 7, 154-161.

(31) Larkin, P. J. Infrared and Raman Spectroscopy; Principles and Spectral Interpretation; Elsevier: Waltham, MA, 2011.

(32) Peng, Y.; Gardner, D. J.; Han, Y.; Kiziltas, A.; Cai, Z.; Tshabalala, M. A. Influence of Drying Method on the Material Properties of Nanocellulose I: Thermostability and Crystallinity. Cellulose 2013, 20, 2379-2392.

(33) Bakirtzis, D.; Delichatsios, M. A.; Liodakis, S.; Ahmed, W. Fire Retardancy Impact of Sodium Bicarbonate on Ligno-Cellulosic Materials. Thermochim. Acta 2009, 486, 11-19.

(34) Mi, Q.-y.; Ma, S.-r.; Yu, J.; He, J.-s.; Zhang, J. Flexible and Transparent Cellulose Aerogels with Uniform Nanoporous Structure by a Controlled Regeneration Process. ACS Sustainable Chem. Eng. 2016, 4, 656-660.

(35) Shi, J.; Lu, L.; Guo, W.; Sun, Y.; Cao, Y. An EnvironmentFriendly Thermal Insulation Material from Cellulose and Plasma Modification. J. Appl. Polym. Sci. 2013, 130, 3652-3658.

(36) Kunič, R. Forest-Based Bioproducts Used for Construction and Its Impact on the Environmental Performance of a Building in the Whole Life Cycle. In Environmental Impacts of Traditional and 
Innovative Forest-Based Bioproducts; Kutnar, A., Muthu, S. S., Eds.; Springer: Singapore, 2016; pp 173-204.

(37) Wang, G.; Chen, X.; Liu, P.; Bai, S. Flame-Retardant Mechanism of Expandable Polystyrene Foam with a Macromolecular Nitrogen-Phosphorus Intumescent Flame Retardant. J. Appl. Polym. Sci. 2017, 134 (1), 44356.

(38) Dou, B.; Li, J.; Wang, Y.; Wang, H.; Ma, C.; Hao, Z. Adsorption and Desorption Performance of Benzene Over Hierarchically Structured Carbon-Silica Aerogel Composites. J. Hazard. Mater. 2011, 196, 194-200.

(39) Li, Z.; Gong, L.; Li, C.; Pan, Y.; Huang, Y.; Cheng, X. Silica Aerogel/Aramid Pulp Composites with Improved Mechanical and Thermal Properties. J. Non-Cryst. Solids 2016, 454, 1-7.

(40) Zhong, L.; Chen, X.; Song, H.; Guo, K.; Hu, Z. Highly Flexible Silica Aerogels Derived from Methyltriethoxysilane and Polydimethylsiloxane. New J. Chem. 2015, 39, 7832-7838. 